\title{
SATISFAÇÃO E O EMPREGO NO SISTEMA ÚNICO DE SAÚDE NA ÁREA DE SAÚDE BUCAL
}

\author{
Satisfaction and employment on Brazilian Health System in Oral Health
}

\author{
Adriana Cristina Oliva Costa \\ ClÉa Adas Saliba Garbin \\ Gabriella Barreto Soares
}

Patrick Raphael Vicente Gonçalves

Suzely Adas Saliba Moimaz

\section{Resumo}

O objetivo do estudo foi avaliar as condições de trabalho dos cirurgiões-dentistas do Sistema Único de Saúde (SUS) por meio de sua própria percepção, analisando: tipo de ingresso, regime de trabalho, carga horária, remuneração percebida, existência de Plano de Carreira, Cargos e Salários (PCCS) e satisfação com o emprego público. A amostra foi composta por cirurgiões-dentistas $(\mathrm{n}=83)$, lotados no sistema público, entre os 12 municípios componentes da pesquisa. A coleta de dados deu-se por meio de entrevistas, utilizandose um questionário semiestruturado previamente testado. Observou-se que, dos entrevistados, 19\% não prestaram concurso público para ingressar no SUS. O regime de trabalho estatutário foi mais adotado, atingindo $57 \%$ dos profissionais. Notaram-se jornadas de trabalho distintas, divididas entre 20,30 e 40 horas de trabalho semanais. A maioria dos profissionais (66\%) estava insatisfeita com sua remuneração e notou-se ainda, a ausência do PCCS em 11 municípios, entretanto, a grande maioria (62\%) afirma estar "satisfeita" quanto ao trabalho no sistema público. Embora o PCCS não seja realidade nos municípios estudados, e a maioria dos profissionais mostrarem-se "insatisfeitos" com a remuneração, quase a totalidade dos entrevistados está "satisfeita" com o emprego público no SUS.

Palavras-chave: Cirurgião-Dentista; REMUNERAÇÃO; SATISFAÇÃO.
Doutora do Programa de Pós-graduação em Odontologia Preventiva e Social da Universidade Estadual Paulista "Júlio de Mesquita Filho. E-mail: costa_ol28@hotmail.com

Professora doutora adjunta do Departamento de Odontologia Infantil e Social da Faculdade de Odontologia de Araçatuba da Universidade Estadual Júlio de Mesquita Filho. E-mail: cgarbin@foa.unesp.br.

Mestranda do Departamento de Odontologia Infantil e Social da Faculdade de Odontologia de Araçatuba da Universidade Estadual Júlio de Mesquita Filho. E-mail:gabriella.

Mestrando do Departamento de Odontologia Infantil e Social da Faculdade de Odontologia de Araçatuba da Universidade Estadual Júlio de Mesquita Filho. E-mail: patrick.raphael@ hotmail.com.

Professora doutora titular do Departamento de Odontologia Infantil e Social da Faculdade de Odontologia de Araçatuba da Universidade Estadual Júlio de Mesquita Filho.

E-mail: sasaliba@foa.unesp.br.

\section{Abstract}

The aim this study was to know work conditions of dental surgeon in Brazilian Health System (SUS), by their perception, analyzing: type of ingress, work regime, workload, realized income, existence of Career Plan, Posts and Ages (PCCS) and satisfaction with public employment. The sample was constituted by dental surgeons $(n=83)$ of 12 cities public system from Health Regional Department XV - São José do Rio Preto City - São Paulo State. Data collection was by interviews using a questionnaire. Results point that 19\% of interviewed people didn't take an open competition to ingress on SUS. Statutory scheme of work was more adopted getting $57 \%$ of professionals. Different working days were observed: $57 \%$ is relative to 20 hours, $7 \%$ to 30 hours and 36 hours to 40 hours. About satisfaction with incomes, $66 \%$ of professionals were dissatisfied, observing the absence of PCCS in 11 cities. About satisfaction with public employment, just 5\% said to be "dissatisfied"; 11\% "few satisfied" and the majority $62 \%$ and $22 \%$ affirmed to be "satisfied" and "satisfied so much" respectively. Although PCCS not to be reality in studied cities and the majority of professionals to say "to be dissatisfied" with wage, around totality of interviewed people is "dissatisfied" with public employment on SUS. Keywords: Dental surgeOn; Wage; SATISFACTION. 


\section{INTRODUÇÃO}

$\mathrm{Na}$ sociedade moderna, o trabalho pode ser entendido como uma atividade fundamental do homem, por meio do qual a satisfação das necessidades humanas, diante da natureza e dos outros homens, é mediatizada. O trabalho é, portanto, uma das formas mais importantes de o homem se posicionar como indivíduo único, por ser algo que o completa e dá sentido à vida. Ele também é visto como um dos componentes da felicidade humana, que é tida como resultante da satisfação plena de necessidades psicossociais, do sentimento de prazer e do sentido de contribuição no exercício da atividade profissional. ${ }^{1}$

O Sistema Único de Saúde (SUS), ao longo de sua construção, tem se caracterizado como uma organização geradora de empregos para os profissionais de saúde. Tal afirmativa pode ser justificada pela própria definição deste "Sistema de Saúde", que, alicerçado na Constituição Brasileira, ${ }^{2}$ está conceituado como instituiçãoorganismo de direito público, que reúne os instrumentos para que o Estado brasileiro desenvolva as atividades necessárias para a garantia do direito à saúde da população.

Com as propostas do Sistema Único de Saúde, houve uma reformulação do modelo de atenção, que passou a assumir uma forma regionalizada de organização fundamentada na descentralização. Deste modo, os municípios se tornaram os maiores responsáveis não somente pela execução das políticas públicas, mas também pela contratação dos trabalhadores do SUS ${ }^{3}$

As políticas públicas do Governo Federal, em se tratando de odontologia, formuladas neste novo século, contribuíram de forma ímpar para o crescimento das categorias odontológicas: houve a inclusão das equipes de Saúde Bucal no Programa da Saúde da Família em 2000, com a Portaria ${ }^{\circ}$ 1444,4 e em 2004, com o surgimento da Política Nacional de Saúde Bucal, ${ }^{5}$ que propõe garantir as ações de promoção, prevenção e recuperação da saúde bucal dos brasileiros, entendendo que essa é fundamental para a saúde geral e a qualidade de vida da população.

Visando à integralidade da atenção em saúde à população brasileira, a Política Nacional de Saúde Bucal apresenta como principais linhas de ação: a viabilização da adição de flúor em estações de tratamento de águas de abastecimento público, a reorganização da Atenção Básica e da Atenção Especializada, denotando-se, assim, a necessidade de recursos humanos para a viabilização de tais políticas públicas de saúde.

No entanto, não é suficiente oferecer serviços de saúde para a população se os profissionais não apresentam condições satisfatórias de trabalho. $\mathrm{O}$ trabalho precário é definido classicamente como "a ausência de proteção social do trabalho, isto é, situação em que este é realizado desprovido de certos direitos e benefícios constitucionalmente assegurados", porém, outras variáveis podem se somar a esse conceito, como: baixos vencimentos ou salários e inadequadas condições de trabalho. ${ }^{6}$

A satisfação no trabalho está sujeita a influências de forças internas e externas ao ambiente de trabalho, sendo considerado um fenômeno complexo e de difícil definição, por se tratar de um estado subjetivo que pode variar de pessoa para pessoa. ${ }^{7}$ Desta forma, sabe-se que a satisfação não está associada a um único agente causal, por exemplo, as questões salariais, mas também a outros fatores, como o ambiente, a existência de carreira, dentre outras causas.

Embora grandes avanços tenham ocorrido na atenção odontológica nesses ${ }^{22}$ anos de construção do Sistema Único de Saúde, estudos têm revelado que a Equipe de Saúde Bucal, nas diversas regiões do país, muitas vezes tem se deparado com a situação do "trabalho precário", fato este que fragiliza a integralidade da atenção em saúde. Diante disso, o objetivo deste estudo foi conhecer as condições de trabalho no SUS por meio da percepção dos trabalhadores da área de saúde bucal.

\section{Metodologia}

Trata-se de estudo transversal, tipo inquérito, observacional, quanti-qualitativo, cuja coleta de dados foi feita por meio de observações e entrevistas, por um questionário semiestruturado, composto por questões abertas e fechadas. Essa pesquisa foi realizada nos 12 municípios pertencentes ao Departamento Regional de Saúde DRS XV do Estado de São Paulo. Foi obtida uma listagem no DRS XV - São José do Rio Preto - dos municípios com o número de cirurgiões-dentistas, vinculados à rede pública de serviços de saúde $(\mathrm{n}=172)$ profissionais. Os sujeitos da pesquisa, incluídos no presente estudo, foram os cirurgiõesdentistas do Sistema Único de Saúde, que estavam ativos nos municípios pertencentes à DRS XV, e que concordaram em participar da pesquisa. Como critério de exclusão, considerou-se afastamento do serviço por licença ou aposentadoria e não aceitação em participar do estudo 
Os 12 municípios foram visitados e, do total de 172 cirurgiões-dentistas (CD) existentes, 83 foram entrevistados em seu local de trabalho por um único pesquisador treinado. Para os profissionais que atuavam em mais de um município, foi considerada a entrevista realizada no primeiro município visitado. O estudo foi aprovado pelo Comitê de Ética em Pesquisa da Faculdade de Odontologia de Araçatuba-UNESP, protocolo FOA2008-01660. O questionário elaborado foi testado em estudo piloto e as seguintes variáveis foram incluídas: tipo de ingresso, regime de trabalho, jornada de trabalho, tempo de serviço, existência de Plano de Carreira, Cargos e Salários (PCCS), gênero, faixa etária, ano de graduação, remuneração e satisfação com o emprego público. Os dados coletados foram processados utilizandose o Software Epi Info 2000 versão 3.5.18 e, posteriormente, realizada a estatística descritiva. Para verificação de associação entre satisfação com salário e satisfação com o emprego público, foi utilizado o teste exato de Fisher.

\section{Resultados}

Os participantes da pesquisa eram em sua maioria do sexo feminino (69\%), na faixa etária entre 31 a 50 anos (68\%), e atuavam entre 15 a 20 anos (35\%) no Sistema Público de Saúde. Verificouse, também, que grande parcela da amostra (45\%) graduou-se na década de oitenta, e que após o término do ensino superior, eles relataram ter concluído ou estar cursando pós-graduação nos diferentes níveis, quer seja especialização, quer seja mestrado ou doutorado (57\%). Nota-se, ainda, que a maioria das pós-graduações realizadas 42 (89\%) refere-se às áreas clínicas, e não à área específica de Saúde Coletiva, Saúde pública ou Estratégia da Saúde da Família. (Tabela 1).

Quanto à forma de ingresso ao quadro de funcionários públicos, os dados apontam que $67(81 \%)$ profissionais prestaram concurso público e os demais 16 (19\%) ingressaram sem concurso. Dos profissionais contratados sem concurso público, 12 foram para exercer o cargo de cirurgiãodentista, e 4 por nomeação a fim de ocupar cargo de confiança. No que se refere ao regime de trabalho adotado, o estatutário 47 (57\%) foi o mais frequente nos municípios estudados, embora também se tenha constatado que 30 (36\%) eram regidos pela CLT e 6 (7\%) não sabiam informar (Tabela 2).

A jornada de trabalho observada foi, em sua maioria, de 20 ou 40 horas de trabalho semanal, sendo: de $20 \mathrm{~h}$ para 47 (57\%), e $40 \mathrm{~h}$ para 30 (36\%) sujeitos do estudo (Tabela 2). Dos integrantes da pesquisa, 78 (94\%) exerciam somente a função de cirurgião-dentista, 1 (1\%) coordenador de saúde bucal, 3 (4\%) desempenhavam função de dentista e coordenador e 1 (\%) exercia o cargo de secretário municipal de saúde. Entretanto, muitos desses profissionais não dedicavam o seu exercício profissional, exclusivamente, para o SUS, sendo que 45 (54\%) atuavam no SUS e em consultórios particulares.

Tabela 1 Perfil dos Cirurgiões-dentistas do SUS, segundo gênero, faixa etária, graduação e pós-graduação, de municípios pertencentes ao Departamento Regional de Saúde - DRS XV do Estado de São Paulo, 2011.

\begin{tabular}{|c|c|c|}
\hline \multirow{2}{*}{$\begin{array}{l}\text { Variável } \\
\text { Gênero }\end{array}$} & \multicolumn{2}{|c|}{ Profissionais } \\
\hline & $\mathbf{n}$ & $\%$ \\
\hline Feminino & 57 & 69 \\
\hline Masculino & 26 & 31 \\
\hline Total & 83 & 100 \\
\hline \multicolumn{3}{|l|}{ Faixa etária } \\
\hline 20 a 30 anos & 6 & 7 \\
\hline 31 a 40 anos & 22 & 27 \\
\hline 41 a 50 anos & 35 & 42 \\
\hline 51 a 60 anos & 18 & 22 \\
\hline 61 a 70 anos & 2 & 2 \\
\hline Total & 83 & 100 \\
\hline \multicolumn{3}{|l|}{ Ano e graduação } \\
\hline 1970 a 1979 & 14 & 17 \\
\hline 1980 a 1989 & 37 & 45 \\
\hline 1990 a1999 & 24 & 29 \\
\hline 2000 a 2006 & 8 & 9 \\
\hline Total & 83 & 100 \\
\hline \multicolumn{3}{|l|}{ Pós-graduação } \\
\hline \multicolumn{3}{|l|}{ Nível } \\
\hline Especialização cursando/concluída & 40 & 48 \\
\hline Mestrado cursando/concluída & 6 & 7 \\
\hline Doutorado cursando/concluído & 2 & 2 \\
\hline Nenhuma & 36 & 43 \\
\hline Total & 83 & 100 \\
\hline \multicolumn{3}{|l|}{$\begin{array}{l}\text { Área de atuação da pós- } \\
\text { graduação }\end{array}$} \\
\hline Clínica & 42 & 89 \\
\hline $\begin{array}{l}\text { Saúde Coletiva/Saúde Pública/ } \\
\text { ESF }\end{array}$ & 5 & 11 \\
\hline Total & 47 & 100 \\
\hline
\end{tabular}


Tabela 2 - Ingresso, tempo de trabalho e aspectos contratuais de Cirurgiões-dentistas do SUS, de municípios pertencentes ao Departamento Regional de Saúde - DRS XV do Estado de São Paulo, 2011.

\begin{tabular}{|l|c|c|}
\hline Variáveis & \multicolumn{2}{|c|}{ Profissionais } \\
\hline Tipo de ingresso & $\mathbf{n}$ & $\%$ \\
\hline Com concurso público & 67 & 81 \\
\hline Sem concurso público & 16 & 19 \\
\hline Total & $\mathbf{8 3}$ & $\mathbf{1 0 0}$ \\
\hline Regime jurídico de trabalho & & \\
\hline CLT & 30 & 36 \\
\hline Estatutário & 47 & 57 \\
\hline Não sabia informar & 6 & 7 \\
\hline Total & $\mathbf{8 3}$ & $\mathbf{1 0 0}$ \\
\hline Jornada de trabalho & & \\
\hline $20 \mathrm{~h} /$ semana & 47 & 57 \\
\hline $30 \mathrm{~h} /$ semana & 6 & 7 \\
\hline $40 \mathrm{~h} /$ semana & 30 & 36 \\
\hline Total & $\mathbf{8 3}$ & $\mathbf{1 0 0}$ \\
\hline Tempo de trabalho na instituição & & \\
\hline Até 5 anos & 17 & 21 \\
\hline 5 a 10 anos & 10 & 12 \\
\hline 10 a 15 anos & 12 & 14 \\
\hline 15 a 20 anos & 29 & 35 \\
\hline 20 a 25 anos & 11 & 13 \\
\hline 25 a 30 anos & 4 & 5 \\
\hline 30 a 35 anos & 0 & 0 \\
\hline Total & $\mathbf{8 3}$ & $\mathbf{1 0 0}$ \\
\hline
\end{tabular}

Com relação ao salário dos entrevistados, os valores relatados pelos profissionais com acúmulo de função de cirurgião-dentista e coordenador de saúde bucal foram de $\mathrm{R} \$ 1.250,00, \mathrm{R} \$ 1.900,00$, $\mathrm{R} \$ 3.000,00$ para jornada de $20 \mathrm{~h}$ e $\mathrm{R} \$ 1.600,00$ para jornada de $30 \mathrm{~h}$. Já para os profissionais com a função de cirurgião-dentista, a remuneração variou entre valores inferiores a 2,29 salários mínimos ( $\mathrm{R} \$ 1.250,00)$ até 12,84 salários mínimos ( $\mathrm{R} \$ 7.000,00$ ) para as jornadas de trabalho de 20h, 30h e 40h (Tabela 3). Entretanto, no que diz respeito à satisfação em relação ao salário recebido, os 78 profissionais que exerciam a função de cirurgião-dentista, 52 (66\%) estavam insatisfeitos com a remuneração recebida, dos 3 profissionais com dupla função (coordenador e cirurgiãodentista), encontravam-se insatisfeitos, e somente o profissional que exercia apenas o cargo de coordenador de saúde bucal mostrava-se realizado com a remuneração. Foi observado também neste estudo a ausência do Plano de Carreira Cargos e Salários (PCCS) em onze $(91,66 \%)$ dos municípios integrantes da pesquisa.

Por meio do Teste Exato de Fisher, verificouse que não houve associação estatisticamente significativa $(p=0,3259)$ entre a satisfação profissional com o salário recebido e a satisfação com o emprego no SUS (Tabela 4).

\section{Discussão}

O advento da municipalização da saúde constitui o processo de descentralização do SUS, que se caracteriza como uma fonte geradora de empregos decorrentes do aumento do número de

TABELA 3 - Remuneração de Cirurgiões-dentistas do SUS, de municípios pertencentes ao Departamento Regional de Saúde - DRS XV do Estado de São Paulo, 2011.

\begin{tabular}{|l|c|c|c|c|c|}
\hline \multirow{2}{*}{ Remuneração Percebida } & \multicolumn{2}{|c|}{ Jornada Semanal } & \multicolumn{2}{c|}{ Total } \\
\cline { 2 - 6 } & $\mathbf{2 0 h}$ & $\mathbf{3 0 h}$ & $\mathbf{4 0 h}$ & $\mathbf{n}$ & \multicolumn{1}{c|}{} \\
\hline 545,00 a $1.635,00$ & 10 & & 2 & 12 & 15 \\
\hline $1.635,00$ a $3.270,00$ & 26 & 4 & 8 & 38 & 46 \\
\hline $3.270,00$ a $4.905,00$ & 8 & 2 & 2 & 12 & 14 \\
\hline $4.905,00$ a $6.540,00$ & 3 & & 2 & 5 & 6 \\
\hline $6.540,00$ a $8.175,00$ & & & 10 & 10 & 12 \\
\hline $8.175,00$ a $9.810,00$ & & & 6 & 6 & 7 \\
\hline Total & & & & $\mathbf{8 3}$ & $\mathbf{1 0 0}$ \\
\hline * Salário mínimo R\$ $\mathbf{5 4 5 , 0 0}$ Lei $\mathbf{n}^{\mathbf{0}} \mathbf{1 2 . 3 8 2 / 2 0 1 1}$ DOU $\mathbf{2 8 / 0 2 / 2 0 1 1}$ & & & & \\
\hline
\end{tabular}


postos de trabalho no setor público. O referido crescimento da oferta de trabalho no setor público passa a ter relevância para os cirurgiõesdentistas a partir dos anos 90, onde houve uma maior inserção destes profissionais dentro da rede SUS, modificando o cenário de trabalho odontológico no país. ${ }^{9}$

TABela 4 - Associação entre satisfação com salário e satisfação com emprego público de Cirurgiõesdentistas do SUS, de municípios pertencentes ao Departamento Regional de Saúde - XV do Estado de São Paulo, 2011.

\begin{tabular}{|c|c|c|c|}
\hline $\begin{array}{l}\text { Salário } \\
\text { Emprego }\end{array}$ & Sim & Não & Total \\
\hline Sim & 23 & 47 & 70 \\
\hline Não & 2 & 11 & 13 \\
\hline Total & 25 & 58 & 83 \\
\hline
\end{tabular}

Teste exato de Fisher $p=0,3259$

A mudança no cenário odontológico durante os anos 90 também é marcada por uma inversão do perfil dos cirurgiões-dentistas, onde uma profissão que possuía caráter essencialmente masculino passa a ter predomínio do gênero feminino. Diversos autores, ${ }^{9,10,11,12,13}$ ao analisarem a questão do gênero no exercício da profissão de odontologia no Brasil, têm revelado ser essa área, atualmente, com predomínio do sexo feminino, o que corrobora com os achados do presente estudo, e demonstra que a feminilização da odontologia também ocorre no setor público.

No código de ética odontológico, está bem claro que, no exercício da profissão, o cirurgiãodentista deve estar sempre atualizado. ${ }^{14}$ Este estudo revelou que a maioria dos cirurgiões-dentistas, após a graduação, continuou aperfeiçoandose, assim como os achados de Bastos et al. ${ }^{15}$ Foi observado, no estudo realizado no Rio Grande do Norte, que $66,8 \%$ dos cirurgiões-dentistas entrevistados não possuíam pós-graduação na área de saúde pública, saúde coletiva ou área afim, que os capacitasse para trabalhar na ESF, corroborando com os dados do presente estudo. ${ }^{16}$

O ingresso de trabalho no setor público ocorre somente com a aprovação prévia em concurso, desde a promulgação da Constituição Federal de 1988,2 que de uma forma lógica e objetiva, fechou as portas para o nepotismo e para o "favorecimento político". Entretanto, foi observado que do total de profissionais entrevistados, uma parcela destes indivíduos ingressou no setor público sem concurso prévio, assim como em outros estudos, que também constataram a indicação como forma de contratação no serviço público. ${ }^{17,18}$

O regime de trabalho adotado do tipo estatutário foi mais frequente neste estudo, estando consoante com a $\mathrm{CF} / 88$, que passa a assegurar direitos e benefícios tanto aos "servidores estatutários", regidos por um estatuto, quanto aos "empregados públicos", subordinados às normas de Consolidação das Leis do Trabalho - CLT, até então esquecidos. Está claro, desde a promulgação da $\mathrm{CF} / 88$, que empregados/servidores públicos somente poderão ingressar em cargo ou emprego público com a aprovação prévia em concurso público, embora sejam ressalvadas as nomeações para cargo em comissão que se destinam apenas às atribuições de direção, chefia e assessoramento. ${ }^{2}$

A existência de diversidade de contratos e formas de ingresso caracteriza a precarização das relações de trabalho no SUS. Estudos revelam que profissionais de Saúde Bucal, em distintas regiões do país, vivenciam a situação do "trabalho precário", fato este que fragiliza a integralidade da atenção em saúde. Autores verificaram, ${ }^{19} \mathrm{em}$ uma pesquisa de âmbito nacional, que as formas de contratação, pelos municípios, de profissionais da equipe de saúde bucal eram realizadas por contratos temporários ou prestação de serviços.

Quanto à carga horária relatada pelos profissionais, pode-se observar uma diversidade de horas trabalhadas por semana, embora não exista impedimento legal que fixe a existência de apenas um tipo de jornada. É notório que independente da carga horária semanal, grande parcela dos cirurgiões-dentistas atua também em clínicas particulares, como revelam os dados obtidos, embora alguns destes profissionais trabalhem exclusivamente no SUS, estando de acordo com outros estudos. ${ }^{20 .}$

Como observado por Souza e Roncalli,17 tal fato pode ser justificado aos baixos salários recebidos. Embora o presente estudo não tenha avaliado a questão do cumprimento da carga horária trabalhada, 45 cirurgiões-dentistas relataram trabalhar no SUS e em consultório particular, por considerarem insuficiente o salário percebido no Sistema Público de Saúde e a ausência do Plano de Carreira, Cargos e Salários (PCCS) na maioria dos municípios integrantes da pesquisa.

A ausência do Plano de Carreira, Cargos e Salários ainda é uma realidade em muitos 
municípios brasileiros e, neste estudo, não foi diferente. Vale lembrar que a discussão sobre o tema PCCS-SUS vem ocorrendo por mais de duas décadas e, apesar de existir amparo legal, na Lei Federal 8.142/90, no Art. $4^{\circ}$, inciso VI, que determina a obrigatoriedade da existência do PCCS para todo o sistema de saúde, nas três esferas de governo, a aplicação do mesmo tem sido um grande desafio para os gestores. A implementação seria uma das formas de valorização dos profissionais da saúde no SUS, uma vez que, por meio desse é possível um sistema de carreiras unificadas, baseado na produtividade, no desempenho, na capacitação (educação permanente) e na progressão com a admissão por concurso público, fatores que contribuirão para a valorização dos profissionais de saúde. Conforme Machado, ${ }^{21} \mathrm{o}$ PCCS não deve ser considerado o responsável pelo "engessamento" da gestão nem continuar sendo tratado com tão pouca seriedade pelos gestores públicos. Da mesma forma, é incorreto associar o PCCS à noção de atraso gerencial, à inoperância administrativa, como muitos "modernos administradores" tentam passar. Ao contrário, o PCCS, se bem estruturado e corretamente executado, com a devida flexibilidade que a realidade impõe, é uma garantia de que o trabalhador terá o direito de vislumbrar uma vida profissional ativa, na qual a qualidade técnica e a produtividade sejam variáveis-chave para a construção de um sistema exequível. ${ }^{12}$

Nesta pesquisa, não houve associação estatística entre a satisfação com o salário e a satisfação com o emprego no SUS, afirmativa que corrobora com os resultados obtidos por Nicolielo e Bastos ${ }^{22}$ e Batista et al. ${ }^{23}$. Esses autores também afirmam que a remuneração se apresenta como um fator de motivação para o trabalho, contudo não constitui o principal fator.

Em vista do resultado, sugere-se que a satisfação com o emprego público e não com o salário, neste estudo, também possa estar relacionada com as vantagens adquiridas com o vínculo empregatício, por exemplo, salário fixo mensal, $13^{\circ}$ salário, férias remuneradas, licença saúde, aposentadoria e, principalmente, a estabilidade no emprego público. Além desses fatores, os cirurgiões-dentistas entrevistados, consideram extremamente importante o trabalho desenvolvido no SUS a benefício da população.

\section{Conclusão}

Embora o Plano de Carreira, Cargos e Salários não seja realidade nos municípios estudados, e a maioria dos profissionais se diz "insatisfeita" com a remuneração, quase a totalidade dos entrevistados está "satisfeita" com o emprego público no SUS.

\section{REFERÊNCIAS BIBLIOGRÁFICAS}

1. Gadotti M. Marx, transformar o mundo. São Paulo: FDT, 1991.

2. BRASIL. Constituição da República Federativa do Brasil. Brasília: Senado Federal, 1988.

3. Faria HX, Dalbello-Araújo M. Precarização do trabalho e processo produtivo do cuidado. Mediações 2011;16(1):142-156.

4. BRASIL. Ministério da Saúde. Portaria $n^{\circ}$. 1.444, de 28 de dezembro de 2000: estabelece incentivo financeiro para reorganização da saúde bucal prestada nos municípios por meio do Programa Saúde da Família. Diário Oficial da União, Brasília, p.85, 29 dez. 2000.

5. BRASIL. Ministério da Saúde. Secretaria de Atenção à Saúde. Departamento de Atenção Básica. Coordenação Nacional de Saúde Bucal. Diretrizes da Política Nacional de Saúde Bucal . Brasília: Ministério da Saúde, 2004.

6. Araújo LM. Machado MH, Vitalino HA, Paiva J, Toloza DC. Para subsidiar a discussão sobre a desprecarização do trabalho no SUS. Cadernos RH Saúde 2006;3(1) :163-173.

7. Fraser TM. Human stress, work and job satisfaction: a critical approach. Geneva: International Labour Office, 1983.

8. Centers for Disease Control and Prevention. Epi Info Versão 3.5.1: software para profissionais de saúde. Atlanta: CDC, 2008.

9. Freitas, C. H. S. M. Dilemas no exercício profissional da odontologia: a autonomia em questão. Interface: Comunicação, Saúde, Educação 2007;11 (21): 25-38.

10. Moimaz SAS, Saliba NA, Blanco MRB. A força do trabalho feminino na odontologia, em Araçatuba - SP. J Applied Oral Science 2003;11( 4):301-305.

11. Bengmark D, Nilner M, Rohlin M. Graduates' characteristics and professional situation: a follow-up of five classes graduated from the Malmö model. Swedish Dental Journal 2007;31(3):129-135.

12. Costa SM, Durães SJA, Abreu MHNG. Feminilização do curso de odontologia da Universidade Estadual de Montes Claros. Ciência \& Saúde Coletiva 2010ª $; 15(1): 1865-1873$.

13. Villalba JP, Madureira PR, Barros NF. Perfil profissional do cirurgião-dentista para atuação 
no Sistema Único de Saúde (SUS). Rev Inst Ciênc. Saúde 2009;27( 3):262-268.

14. Conselho Federal de Odontologia. Código de ética odontológica. Rio de Janeiro: CFO, 2006.

15. Bastos JRM, Aquilante AG, Almeida BS, Lauris JRP, Bijella VT. Análise do perfil profissional de cirurgiões-dentistas graduados na Faculdade de Odontologia de Bauru-USP entre os anos de 1996 e 2000. J Applied Oral Science 2003; 11(4): 283-289.

16. Costa RM. Medeiros Júnior A, Costa ICC, PINHEIRO IVA. Processo de trabalho do dentista na estratégia de saúde da família do município de Parnamirim-RN: enfrentando os desafios de um novo modelo de atenção. Rev Odontol Brasil Central 2010b;19( 51): 327-332.

17. Souza TMS, Roncalli AG. Saúde bucal no Programa Saúde da Família: uma avaliação do modelo assistencial. Cadernos de Saúde Pública 2007;23(11): 2727-2739.

18. Lourenço EC, Silva ACB, Meneghin MC, Pereira AC. A inserção de equipes de saúde bucal no Programa Saúde da Família no Estado de Minas Gerais. Ciência \& Saúde Coletiva 2009;14(1):1367-1377.
19. BRASIL. Ministério da Saúde. Coordenação Geral da Política de Recursos Humanos. Agentes institucionais e modalidades de contratação de pessoal no Programa Saúde da Família no Brasil: relatório de pesquisa. Belo Horizonte: Ministério da Saúde, 2002.

20. Lazeris AM, Calvo MCM, Regis Filho GI. A formação de recursos humanos em odontologia e as exigências do setor público: uma contribuição para serviços de saúde públicos e de qualidade. Rev. Odonto Ciência 2007;22( 56):166-176.

21. Machado, M. H. Gestão do trabalho em saúde no contexto de mudanças. Rev. Admin. Publ. 2000;34(4):133-146.

22. Nicolielo J, Bastos JRM. Satisfação profissional do cirurgião dentista conforme tempo de formado. Rev. Fac. Odontol. Bauru 2002;10( 2):69-74.

23. Batista AAV, Vieira MJ, Cardoso NCS, Carvalho GRP. Fatores de motivação e insatisfação no trabalho do enfermeiro. Rev. Esc. Enferm. USP 2005; 93(1): 85-91.

Submetido em: $30-8 / 2012$

Aceito em: 28-11-2012 